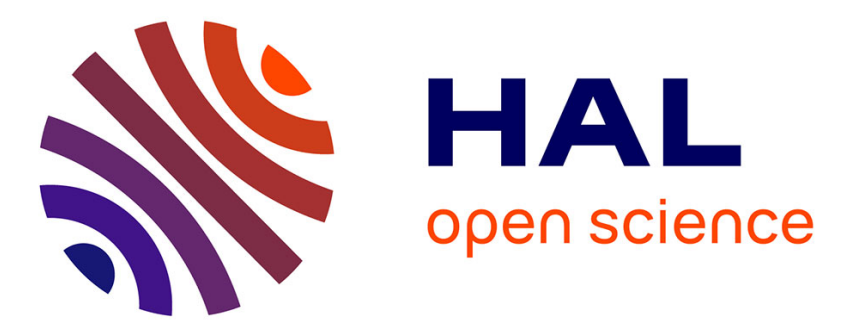

\title{
Expertise in a Hybrid Diagnostic-Recommendation System for SMEs: A Successful Real-Life Application
}

\author{
Sylvain Delisle, Josée St-Pierre
}

\section{To cite this version:}

Sylvain Delisle, Josée St-Pierre. Expertise in a Hybrid Diagnostic-Recommendation System for SMEs: A Successful Real-Life Application. 17th International Conference on Industrial et Engineering Applications of Artificial Intelligence (IEA/AIE), 2004, Ottawa, Canada. hal-01704927

\section{HAL Id: hal-01704927 \\ https://hal.science/hal-01704927}

Submitted on 8 Feb 2018

HAL is a multi-disciplinary open access archive for the deposit and dissemination of scientific research documents, whether they are published or not. The documents may come from teaching and research institutions in France or abroad, or from public or private research centers.
L'archive ouverte pluridisciplinaire HAL, est destinée au dépôt et à la diffusion de documents scientifiques de niveau recherche, publiés ou non, émanant des établissements d'enseignement et de recherche français ou étrangers, des laboratoires publics ou privés. 


\title{
Expertise in a Hybrid Diagnostic-Recommendation System for SMEs: A Successful Real-Life Application
}

\author{
Sylvain Delisle ${ }^{1} \&$ Josée St-Pierre ${ }^{2}$ \\ Institut de recherche sur les PME \\ Laboratoire de recherche sur la performance des entreprises \\ Université du Québec à Trois Rivières \\ 1: Département de mathématiques et d'informatique \\ 2: Département des sciences de la gestion \\ C.P. 500, Trois-Rivières, Québec, Canada, G9A 5H7 \\ Phone: 1-819-376-5011+3832 \\ Fax: 1-819-376-5185 \\ Email: \{sylvain_delisle, josee_st-pierre\}@uqtr.ca \\ Web: www.uqtr.ca/ $\{\sim$ delisle, dsge\}
}

\begin{abstract}
We describe a hybrid expert diagnosis-recommendation system we have developed for SMEs. The system is fully implemented and operational, and has been successfully put to use on data from actual SMEs. Although the system is packed with knowledge and expertise, it was not implemented with "traditional" symbolic AI techniques. We explain why and discuss how the system relates to expert systems, decision support systems, and AI. We also report on an experimental evaluation and identify ongoing and future developments.
\end{abstract}

\section{Introduction}

In this application-oriented paper, we briefly describe a hybrid expert system we have developed for SMEs. Using a benchmarking approach [1,23], our system performs a multidimensional evaluation of a SME's production and management activities, and assesses the results of these activities in terms of productivity, profitability, vulnerability and efficiency. This system is fully implemented and operational, and has been put to use on actual data from some 500 SMEs from Canada, USA, and France. By academic standards, it is clearly a successful real-life application.

What is peculiar though, especially from a knowledge-based systems perspective, is the fact that although the PDG system is packed with knowledge and expertise on SMEs, it has not been implemented with "traditional" symbolic Artificial Intelligence (AI) techniques. However, the PDG system certainly qualifies as a hybrid diagnosticrecommendation expert system. In the following sections, we provide further details on the PDG system and how it relates to knowledge-based and expert systems, as well as to decision support systems. We also identify aspects of the PDG that will benefit from the addition of AI techniques in current and future developments.

Our work takes place within the context of the Research Institute for SMEs. The Institute's core mission is to support fundamental and applied research to foster the advancement of knowledge on small and medium-sized enterprises (SMEs) to con- 
tribute to their development. Our lab, the LaRePE (LAboratoire de REcherche sur la Performance des Entreprises: www. uqtr.ca/inrpme/larepe/), is mainly concerned with the development of scientific expertise on the study and modeling of SMEs' performance, including a variety of interrelated subjects such as finance, management, information systems, production, technology, etc. All research projects carried out at the LaRePE involve both theoretical and practical aspects, always attempting to provide practical solutions to real problems confronting SMEs, often necessitating in-field studies.

\section{Expert Systems, Decision Support Systems, and AI}

Since results produced by the PDG system can be used by SME owners to make decisions regarding the management of their business, the PDG system can be regarded as a decision support system (DSS) $[16,20]$ that uses benchmark data to compare SMEs against their peers - a group of peers constitutes a reference group for benchmarking purposes. We now look at various aspects of expert systems (ES), DSS, and AI that are relevant to the conceptual background of the PDG system before we get into the specifics of that system in Section 3.

DSSs exist to assist someone in making a choice, rendering a judgement, or drawing a conclusion. Their operation is subordinated to the human user, who remains central to and in control of the decision-making process. Because ESs however emulate the human reasoning process, they typically operate automatically until they reach a conclusion which is then communicated to their user. This observation is supported by Forgionne et al. [3], who adopt the same fundamental division of decision support mechanisms into autonomous or assistive systems. ESs and DSSs are applied, driven by exigencies encountered in the real world, rather than a working-out of some conception of a theoretical model.

Although ESs and other AI techniques are often used in DSSs, we are unaware of any implementation in which a DSS assists an ES save that proposed by Plenert [11], which would use a DSS for a period of time to capture decisions which could subsequently be codified to produce the rules of an ES. A quite substantial literature on the other hand discusses the use of AI in DSS. While it would be generally agreed that symbolic ES approaches are the AI techniques used most often in DSSs, it should be noted that non-symbolic decision-making methodologies such as neural nets [5] and genetic algorithms [22] are also employed.

Houben et al. [4] argue for the use of a knowledge-based DSS to make strategic decisions in DSSs. Although their system for identifying and assessing organizational strengths, weaknesses, opportunities and threats ('SWOT') appears not to have actually been put to use, the authors report that the "general opinion of the experts about the validity of the system was quite positive and encouraging" and that "only about $10 \%$ of the output of the system was doubted by the experts". Rosca and Wild [13] survey the study and use of business rules, which are natural language statements expressing the policies of an enterprise in a manner akin to the antecedent-consequent syntax commonly used in an expert system. Business rules thus separate out the rulebase from the ES and treat it as an object of study in itself. 
Only limited research has been done into the particular area that interests us, the use of ESs in DSSs which benchmark small and medium-sized industrial enterprises. Muhlemann et al. [9] report on the adoption and evolution of a generic production management DSS in two SMEs. They found that the system acted as a "change agent", evolving to encompass decisions of greater scope, and that this evolution was facilitated by the good production management practices embodied in the software. Price et al. [12] describe a DSS for manufacturing enterprises which has the goal of supporting more frequent changes in corporate strategy, an emerging business requirement the authors identify. The nature and number of DSS inputs approximate those involved in benchmarking efforts.

Levy and Powell [6] study strategies for SME information systems. One of the main benefits identified of adopting strategic information systems is "obtaining information to manage the business more effectively and competitively", the goal of DSSs. The same authors [7] also found that although many of the lessons learned in large firms remain valid for small ones, there are a number of key differences, such as a dependence on external environment and the absence of formal information system departments in SMEs. These differences are pertinent to DSSs because they presume the existence in the organization of an information system able to produce operational data on which decisions are based.

\section{SME Performance Evaluation with the PDG System}

\subsection{An Overview of the System}

The PDG system, which runs on computers located in our lab, evaluates a SME from an external perspective and on a comparative basis in order to produce a diagnosis of its performance and potential, complemented with relevant recommendations - the project started in 1997 and the current version has been in production for 2 years. The PDG system is a hybrid diagnostic-recommendation system as it not only identifies the evaluated SME's weaknesses, but it also makes suggestions on how to address these weaknesses in order to improve the SME's performance. An extensive (18page) questionnaire is used to collect relevant information items on the SME to be evaluated, along with the financial statements of the last five years. Data extracted from the questionnaire and the financial statements is computerized and fed into the system. The latter performs a multidimensional evaluation in approximately 3 minutes by contrasting the particular SME with an appropriate reference group of SMEs for which we have already collected relevant data; this is the crux of the benchmarking process. The output is a detailed report in which 28 management practices (concerning human resources management, production systems and organization, market development activities, accounting, finance and control tools), 20 results indicators and 22 general information items are evaluated, leading to 14 recommendations on short term actions the evaluated SME could undertake to improve its overall performancedetails of the main computations involved during benchmarking are presented in [18]. For instance, here is an example recommendation on human resources results: "Your overall effectiveness at managing human resources is comparable to that of your ref- 
erence group. You should pay attention to why certain managerial jobs have a high rate of voluntary departure, with the objective of lowering hiring and training costs".

As shown in Figure 1, the PDG expert diagnosis system is connected to an Oracle database which contains all the relevant data for benchmarking purposes. The PDG reports are constantly monitored by a team of multidisciplinary human experts in order to ensure that recommendations are valuable for the entrepreneurs. This validation phase, which always takes place before the report is sent to the SME, is an occasion to make further improvements to the PDG system, whenever appropriate. It is also a valuable means for the human experts to update their own expertise on SMEs. Figure 1 also shows that an intermediary partner is part of the process in order to guarantee confidentiality: nobody in our lab knows to what companies the data are associated.

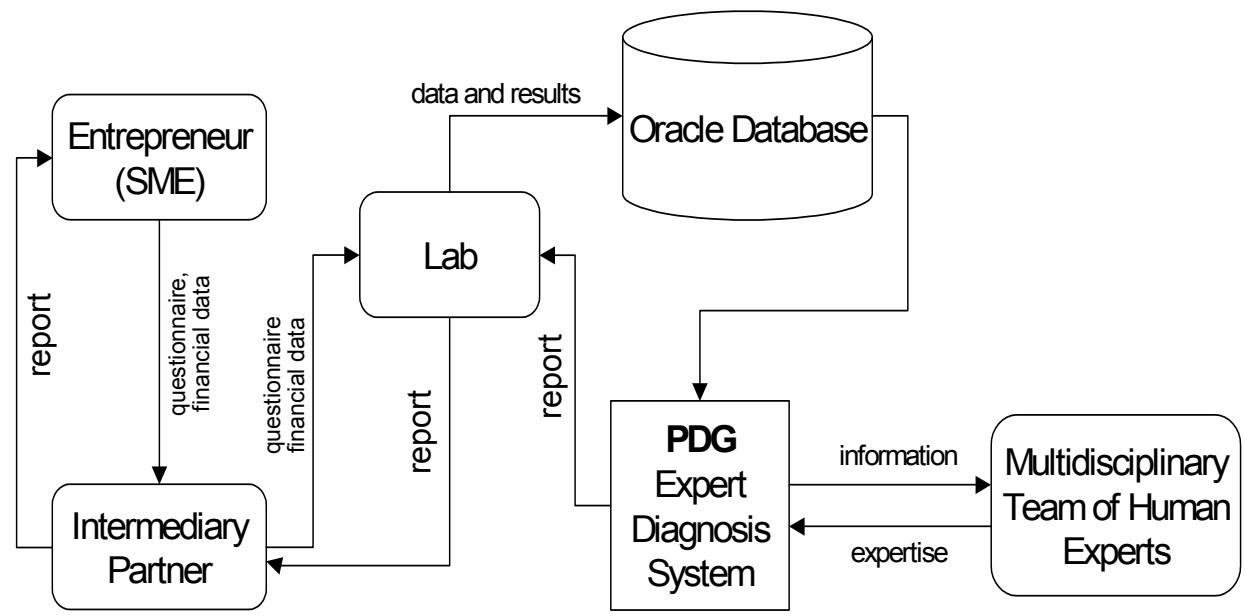

Fig. 1. The PDG system: evaluation of SMEs, from an external perspective and on a comparative basis, in order to produce a diagnosis of their performance and potential

As far as we know, our system is unique. A somewhat similar system is presented in [2]. However, their system was especially developed for SMEs that produce goods in relatively small volumes and in batch. It is based on a specific benchmark focusing on manufacturing and assembly processes. Moreover, the system they describe is mostly semi-automatic (if not mostly manual), whereas ours is entirely automatic, let alone a final revision of the final wording of the main recommendations which usually takes between five to ten minutes.

\subsection{Some Details on the System}

Besides an Oracle database, the PDG uses the SAS statistical package, and Microsoft Excel (see Figure 2 next page). The system's expertise is located in the questionnaire and the benchmarking results interpretation module. The first version of the questionnaire was developed by a multidisciplinary team of researchers in business strategy, human resources, information systems, industrial engineering, logistics, marketing, 
economics, and finance. The questionnaire development team was faced with two important challenges: 1) find a common language (a shared ontology) that would allow researchers to understand each other and, at the same time, would be accessible to entrepreneurs when answering the questionnaire, and 2) identify long-term performance indicators for SMEs, as well as problem indicators, while keeping contents to a minimum since in-depth evaluation was not adequate.

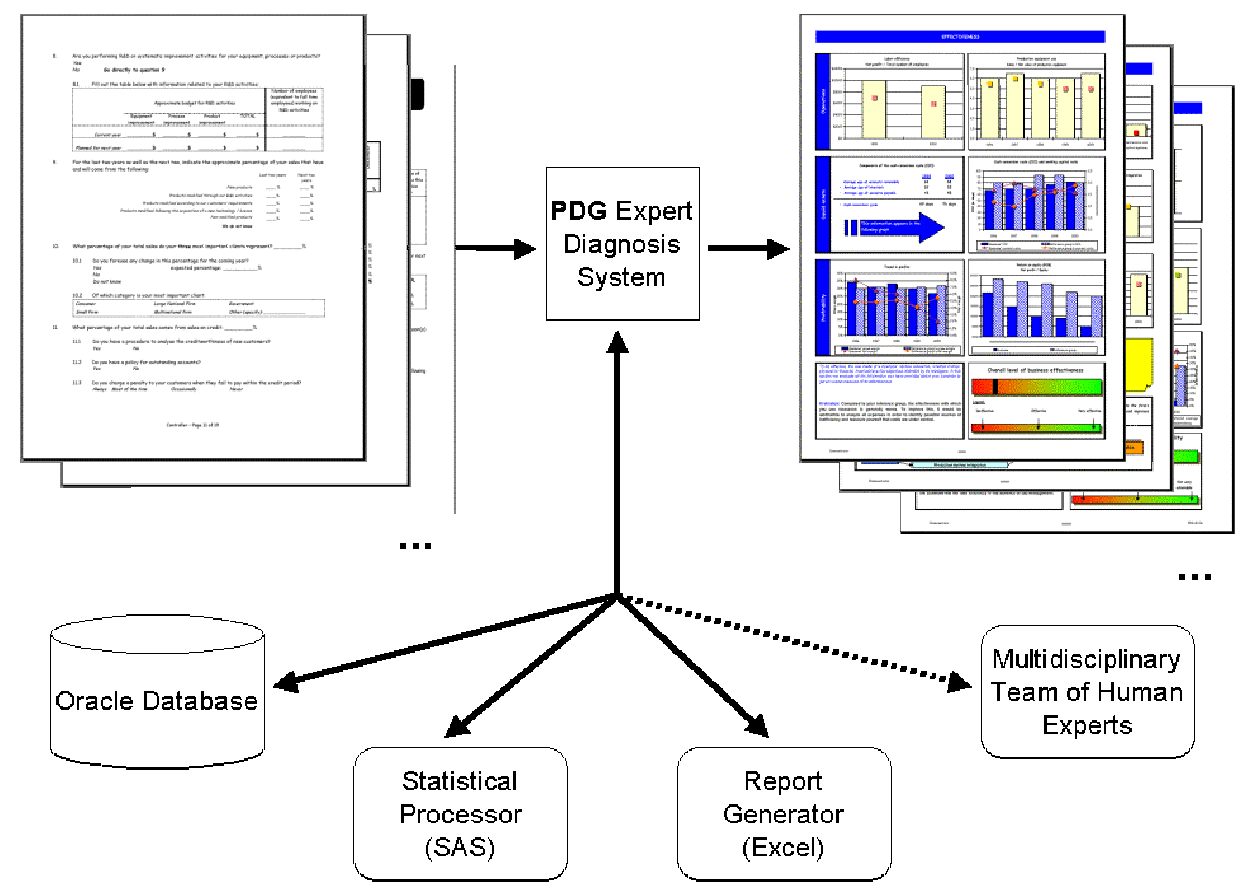

Fig. 2. The PDG system and its main components: from a broad-coverage questionnaire and financial statements to a detailed benchmarking evaluation and recommendations.

The team was able to meet these two goals by the assignment of a "knowledge integrator" role to the project leader. During the 15-month period of its development, the questionnaire was tested with entrepreneurs in order to ensure that it was easy to understand both in terms of contents and question formulation, and report layout and information visualization. All texts were written with a clear pedagogical emphasis since the subject matter was not all that trivial and the intended readership was quite varied and heterogeneous. Several prototypes were presented to entrepreneurs and they showed a marked interest for graphics and colours.

The researchers' expertise was precious in the identification of vital information that would allow the PDG system to rapidly produce a general diagnosis of any manufacturing SME. The diagnosis also needed to be reliable and complete, while being comprehensible by typical entrepreneurs. This was pioneering research work that the whole team was conducting. Indeed, other SME diagnosis systems are generally financial and based on valid quantitative data - see $[8,10,21]$ for examples of expert 
systems in finance. Each expert had to identify practices, systems, or tools that had to be implemented in a manufacturing SME to ensure a certain level of performance. Then, performance indicators had to be defined in order to measure to what extent these individual practices, systems, or tools were correctly implemented and allowed the enterprise to meet specific goals - the relationship between practices and results is a distinguishing characteristic of the PDG system. Next, every selected performance indicator was assigned a relative weight by the expert and the knowledge integrator. This weight is used to position the enterprise being diagnosed with regard to its reference group, thus allowing the production of relevant comments and recommendations. The weight is also used to produce a global evaluation that will be displayed in a synoptic table. Contrary to many performance diagnostic tools in which the enterprise's information is compared to norms and standards (e.g. [8]), the PDG system evaluates an enterprise relative to a reference group selected by the entrepreneur. Research conducted at our institute seriously questions this use of norms and standards: it appears to be dubious for SMEs as they simply are too heterogeneous to support the definition of reliable norms and standards.

\begin{tabular}{|c|c|c|}
\hline Scale variable & Binary variable & $\begin{array}{c}\text { Continuous (numerical) } \\
\text { variable }\end{array}$ \\
\hline $\begin{array}{c}\text { if } \mathrm{SME}>=(1.25 \times \mathrm{MEA}) \\
\text { then } \mathrm{CODE}=4\end{array}$ & $\begin{array}{c}\text { if } \mathrm{SME}=1 \text { and } 10 \% \text { of } \mathrm{RG}=1 \\
\text { then } \mathrm{CODE}=4\end{array}$ & $\begin{array}{c}\text { if SME }>=(1.25 \times \text { MED }) \\
\text { then CODE }=4\end{array}$ \\
\hline $\begin{array}{c}\text { if SME }>=(1.10 \times \mathrm{MEA}) \\
\text { then CODE }=3\end{array}$ & $\begin{array}{c}\text { if } \mathrm{SME}=1 \text { and } 25 \% \text { of } \mathrm{RG}=1 \\
\text { then } \mathrm{CODE}=3\end{array}$ & $\begin{array}{c}\text { if SME }>=(1.10 \times \text { MED }) \\
\text { then CODE }=3\end{array}$ \\
\hline $\begin{array}{c}\text { if } \mathrm{SME}>=(1.00 \times \mathrm{MEA}) \\
\text { then CODE }=2\end{array}$ & $\begin{array}{c}\text { if } \mathrm{SME}=1 \text { and } 50 \% \text { of } \mathrm{RG}=1 \\
\text { then } \mathrm{CODE}=2\end{array}$ & $\begin{array}{c}\text { if } \mathrm{SME}>=(1.00 \times \mathrm{MED}) \\
\text { then } \mathrm{CODE}=2\end{array}$ \\
\hline $\begin{array}{c}\text { if } \mathrm{SME}>=(0.90 \times \mathrm{MEA}) \\
\text { then } \mathrm{CODE}=1\end{array}$ & $\begin{array}{c}\text { if } \mathrm{SME}=1 \text { and } 75 \% \text { of } \mathrm{RG}=1 \\
\text { then } \mathrm{CODE}=1\end{array}$ & $\begin{array}{c}\text { if SME }>=(0.90 \times \text { MED }), \\
\text { then CODE = } 1\end{array}$ \\
\hline $\begin{array}{c}\text { if SME }>=(0.75 \times \mathrm{MEA}) \\
\text { then CODE }=0\end{array}$ & $\begin{array}{c}\text { if } \mathrm{SME}=1 \text { and } 90 \% \text { of } \mathrm{RG}=1 \\
\text { then } \mathrm{CODE}=0\end{array}$ & $\begin{array}{c}\text { if SME }>=(0.75 \times \text { MED }) \\
\text { then CODE }=0\end{array}$ \\
\hline $\begin{array}{c}\text { Example: participative } \\
\text { management }\end{array}$ & $\begin{array}{c}\text { Example: remuneration } \\
\text { plan }\end{array}$ & Example: fabrication cost \\
\hline
\end{tabular}

Table 1. Some aspects of the representation of expertise within the PDG system with performance indicators implemented as variables. This table shows three (3) variables: one scale variable (participative management), one binary (remuneration plan), and one continuous numerical (fabrication cost). Legend: $\mathrm{SME}=$ variable value for the evaluated enterprise; MEA = mean value of the variable in the reference group; $\mathrm{RG}=$ reference group; $\mathrm{MED}=$ median value of the variable in the reference group; CODE = resulting code for the evaluated enterprise.

Performance indicators are implemented as variables in the PDG system-more precisely in its database, and in the benchmarking results interpretation module (within the report production module). These variables are defined in terms of three categories: 1) binary variables, which are associated with yes/no questions; 2) scale variables, which are associated with the relative ranking of the enterprise along a 1 to 4 or a 1 to 5 scale, depending on the question; and 3) continuous (numerical) variables, which are associated with numerical figures such as the export rate or the training budget. Since variables come in different types, they must also be processed differently at the statistical level, notably when computing the reference group used for benchmarking purposes. In order to characterize the reference group with a single value, a central tendency measure that is representative of the reference group's set of 
observations is used. Depending on the variable category and its statistical distribution, means, medians, or percentages are used in the benchmarking computations. Table 1 (previous page) shows an example of how the evaluated enterprise's results are ranked and associated with codes that will next be used to produce the various graphics in the benchmarking report.

AI appears in the PDG software primarily as an embedded ES whose rules are implemented as formulae attached in the cells of an Excel spreadsheet that composes the PDG report. It is worth noting that antecedents, if-clauses, in these rules are not parameterized only for client instance data, as is the practice in one class of ES. Instead, both instance data (the data evaluated) and peer group data (the evaluation context) can be parameters. Thus rules in the PDG system have the general form "if clientSME is $20 \%$ less than group_mean ..." where client-SME and group_mean are variables. Many ES consequents, then-clauses, use meaningful variable names and are structured so their meaning is fairly straightforward to the reader. Thus, the rule example above might have the consequent, “... then feed_stock is incorrect". Readable rules make it easier to implement the explanation facilities commonly found in ESs. A PDG rule consequent operates indirectly: its execution does nothing except concatenate an appropriate string from short text fragments. The content of that string is the counterpart of the traditional rule consequent, detailing a course of action to be carried out, not by the system, but by the benchmarking report reader. Finally, with respect to the explanation facility of traditional ES, one might say that when read together, the evaluation and recommendation paragraphs produced by PDG rules are self-explanatory; that is, together they provide their own explanation. Implementing an ES as cell formulae in Excel is not the most obvious way to do it, and the motivation for this fairly unusual approach is further explained in the next section.

\section{Knowledge Engineering Aspects}

A good deal of multi-domain expertise and informal knowledge engineering was invested into the design of the PDG system. In fact, at the early stage of the project, it was even hoped that a traditional expert-system approach would apply naturally to the task we were facing. Using the Visual Rule Studio expert system shell, a prototype was in fact developed for a subset of the full system dealing only with human resources. However, the knowledge acquisition, knowledge modelling, and knowledge validation/verification phases $[14,15]$ were too demanding in the context of our resources constraints, especially in the context of a multidisciplinary domain such as that of SME for which little formalized knowledge exists. Indeed, many people were involved, all of them in various specialization fields, and with various backgrounds (researchers, graduate students, research professionals, entrepreneurs). The development of a truly multidimensional performance evaluation scheme especially tailored to SMEs was, and still is, a quite demanding and challenging endeavour.

One of the main difficulties that hindered the development of the prototype expert system was the continuous change both the questionnaire and the benchmarking report were undergoing during the first three years of the project. At the same time the research team was trying to develop a multidimensional model of SME performance 
evaluation, users' needs had to be considered, software development had to be carried out, and evaluation reports had to be produced for participating SMEs. This turned out to be a rather complicated situation. The human-resources prototype was developed (with the expert system shell) in parallel with the full system version (which was based mainly on database technology). The project leader's knowledge engineer role was very difficult since several experts from different domains were involved and the extraction and fusion of these various fields of expertise had never been done before. Despite the experts' valuable experience, knowledge, and good will, they had never been part of a similar project before. The modelling of such rich, complex, and vast information was an entirely new challenge both scientifically and technically. Indeed, because of their heterogeneous nature, and contrary to large enterprises, SMEs are much more difficult to model and evaluate. Finally, a comparison between the human-resources results produced by the prototype with those produced by the full system revealed that the latter were better.

These important considerations and difficulties, not mentioning the consequences they had on the project's schedule and budget, lead to the abandon of the expert system approach in favour of the database approach. We had to make a rational decision based on our experience, but also on the well-documented fact that multi-domain, multi-expert knowledge acquisition and modelling constitutes a great challenge. Yet another factor that had great influence on our design decisions was the fact that the project started out on paper as a questionnaire, which led naturally to database building and use of all the database-related techniques and technology.

\section{Experimental Evaluation}

A recent experimental evaluation [17] was performed in 2002 on the inventory of reports produced to that date for 307 SMEs, 258 first-time and 49 second-time users. The authors hypothesized that the use of benchmarking would have a positive effect on enterprise operational and financial outcomes, and tested this by comparing the results involved for the first-time and second-time populations as reported in their profiles. Although not all differences are significant, non-parametric statistical analysis ( $t$ - and $\mathrm{z}$-scores) showed general support for the hypothesis. Further examination of the analysis indicated that the 49 second-time users "showed marked improvement in their financial performance from the first to second year". An attempt was then made to gain insight into the relationships between the factors in play using structural equation modelling (partial-least-squares). It suggested that benchmarking has a positive effect on operational performance $(\gamma=.13)$ and financial performance $(\gamma=.08)$, and tends to lead to the implementation of best practices $(\gamma=.16)$. The authors explain the lower gamma for financial performance as reflecting the time required for increases in productivity to show up on the bottom line. 


\section{Conclusion: Toward an Even More Intelligent PDG System}

We have briefly presented a fully implemented expert diagnostic system which evaluates on a benchmarking basis the performance of SMEs. The PDG system has been in use for several years and has gone through a constant and quite challenging evolution in order to meet both the needs of SME-oriented research (numerous research projects have used the system so far) and the production of benchmarking reports for hundreds of SMEs. The current version of the PDG system is not implemented with traditional AI techniques, e.g. knowledge base of symbolic rules and facts, inference engine, etc. Instead, the full system ended up as knowledge-packed system built on database technology that allows it to produce outputs that only a human expert, or in fact several human experts in different domains, would be able to produce in terms of diagnosis and recommendation quality. The output report contains mostly coloured diagrams and simple explanations that are formulated in plain English (or French) so that SMEs entrepreneurs can easily understand it. The system also uses some conventional techniques, e.g. the comments produced in the output report are generated via a templatebased approach, an early technique used in natural language processing.

Our system is now at a stage where we can now consider the introduction of AI techniques in new developments. Here are three examples. 1) The huge number of database attributes and statistical variables manipulated in the system is overwhelming. A conceptual taxonomy, coupled with an elaborated data dictionary, has now become a necessary addition. This work is in progress. 2) Augmenting the PDG system with case-based reasoning seems a promising avenue. Evaluation of the problem at hand could be facilitated if it were possible to establish relationships with similar problems (cases) already solved before-e.g. see [19]. Determining the problems' salient features to support this approach would also offer good potential to lessen the users' burden during the initial data collection phase. This is part of our future work. 3) The development of data warehouses and data mining algorithms to facilitate statistical processing of data and extend knowledge extraction capabilities is a priority. This is the main focus of our current work. Indeed, we are about to activate a new data warehouse and start our first experimentations with data mining techniques. Data warehousing has many practical uses in our SME-oriented context and it will, along with data mining techniques, positively affect the way researchers use the rich data we have collected and continue to collect on SMEs. We hope to significantly extend our knowledge on SMEs, and further improve our evaluation model of SME performance.

\section{References}

1. Cassell C., S. Nadin \& M.Older Gray (2001), "The Use and Effectiveness of Benchmarking in SMEs", Benchmarking: An International Journal, 8(3), 212-222.

2. Denkena B., R. Apitz \& C. Liedtke (2003), "Knowledge-Based Benchmarking of Production Performance", Proc. of the First International Conf. on Performance Measures, Benchmarking and Best Practices in the New Economy (Business Excellence '03), Guimaraes (Portugal), 10-13 June 2003, 166-171.

3. Forgionne G., R. Kohli \& D. Jennings (2002), “An AHP Analysis of Quality in AI and DSS Journals", International Journal of Management Science, 30, 171-183. 
4. Houben G., K. Lenie \& K. Vanhoof (1999), “A Knowledge-Based SWOT-Analysis System as an Instrument for Strategic Planning in Small and Medium Sized Enterprises", Decision Support Systems, 26(2), 125-135.

5. Leung Y.W. \& J.-Y. Mao (2003), "Providing Embedded Proactive Task Support for Diagnostic Jobs: A Neural Network-Based Approach", Expert Systems with Applications, 25(2), 255-267.

6. Levy M. \& P. Powell (2000), "Information Systems Strategy for Small and Medium Sized Enterprises: An Organisational Perspective", The Journal of Strategic Information Systems, 9(1), 63-84.

7. Levy M., P. Powell \& R. Galliers (1999), "Assessing Information Systems Strategy Development Frameworks in SMEs", Information \& Management, 36(5), 247-261.

8. Matsatsinis N.F., M. Doumpos \& C. Zopounidis (1997), "Knowledge Acquisition and Representation for Expert Systems in the Field of Financial Analysis", Experts Systems with Applications, 12(2), 247-262.

9. Muhlemann A., D. Price \& M. Afferson (1995), "A Computer Based Approach for Enhancing Manufacturing Decision Making in Smaller Manufacturing Enterprises: A Longitudinal Study", International Journal of Management Science, 23(1), 97-107.

10. Nedovic L. \& V. Devedzic (2002), "Expert Systems in Finance-A Cross-Section of the Field", Expert Systems with Applications, 23, 49-66.

11. Plenert G. (1994), "Improved Decision Support Systems Help to Build Better Artificial Intelligence Systems", Kybernetes, 23(9), 48-54.

12. Price D., R. Beach, A. Muhlemann, J. Sharp \& A. Paterson (1998), "A System to Support the Enhancement of Strategic Flexibility in Manufacturing Enterprises", European Journal of Operational Research, 109, 362-376.

13. Rosca R. \& C. Wild (2002), "Towards a Flexible Deployment of Business Rules", Expert Systems with Applications, 23, 385-394.

14. Santos J., Z. Vale \& C. Ramos (2002), "On the Verification of an Expert System: Practical Issues", Lecture Notes in Artificial Intelligence \#2358, 414-424.

15. Schreiber G., H. Akkermans, A. Anjewierden, R. de Hoog, N. Shadbolt, W. Van de velde \& B. Wielinga (2002), Knowledge Engineering and Management: The Common KADS Methodology, MIT Press.

16. Shim J.P., M. Warkentin, J.F. Courtney, D.J. Power, R. Sharda \& C. Carlsson (2002), "Past, Present, and Future of Decision Support Technology", Decision Support Systems, 33, 111126.

17. St-Pierre J., L. Raymond \& E. Andriambeloson (2002), "Performance Effects of the Adoption of Benchmarking and Best Practices in Manufacturing SMEs", Proc. of the Conf. on Small Business and Enterprise Development, The University of Nottingham (UK), 15-16 April 2002.

18. St-Pierre J. \& S. Delisle (2004), “An Expert Diagnosis System for the Benchmarking of SMEs' Performance", Benchmarking-An International Journal, Emerald, 11(5-6), to appear.

19. Stamelos I. \& I. Refanidis (2002), "Decision Making Based on Past Problem Cases", Lecture Notes in Artificial Intelligence \#2308, 42-53.

20. Turban E. \& J.E. Aronson (2001), Decision Support Systems and Intelligent Systems, Prentice Hall.

21. Wagner W.P., J. Otto \& Q.B. Chung (2002), "Knowledge Acquisition for Expert Systems in Accounting and Financial Problem Solving", Knowledge-Based Systems, 15, 439-447.

22. Wong M.L. (2001), "A Flexible Knowledge Discovery System Using Genetic Programming and Logic Grammars", Decision Support Systems, 31(4), 405-428.

23. Yasin M.M. (2002), "The Theory and Practice of Benchmarking: Then and Now", Benchmarking: An International Journal, 9(3), 217-243. 